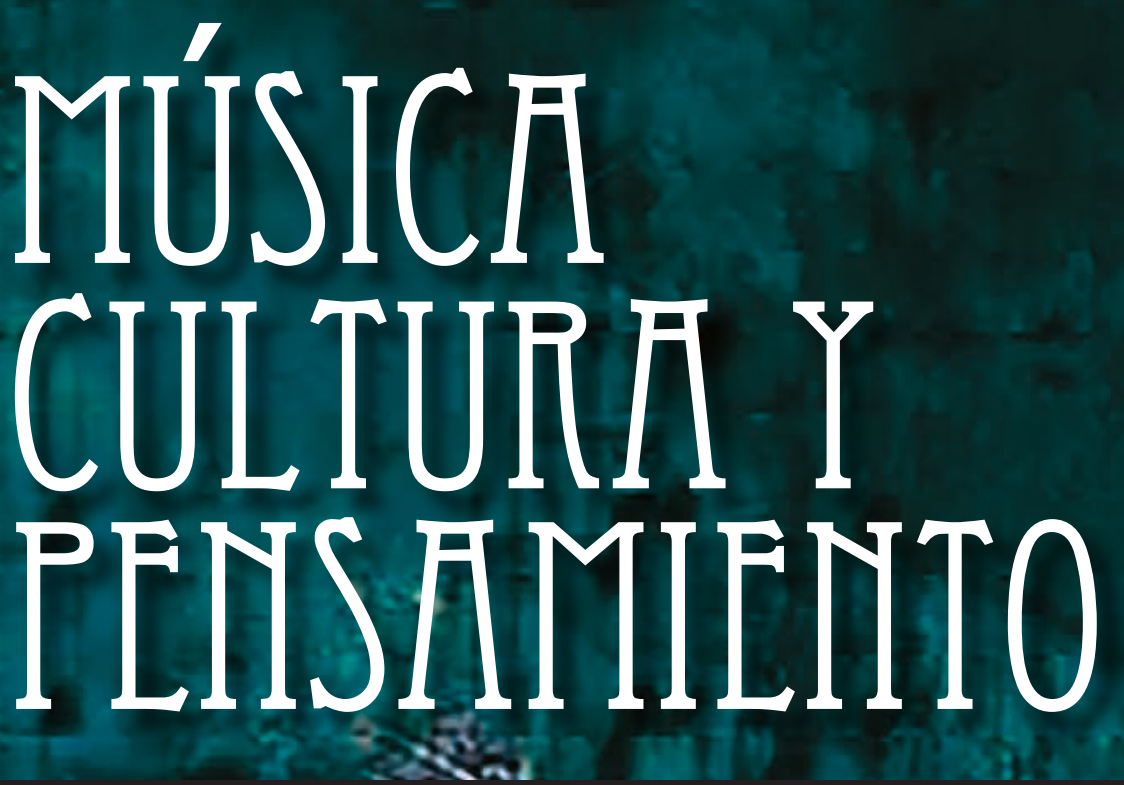

Revista de investigación de la Facultad de Educación y Artes del Conservatorio del Tolima
VOL. VII N.` 7 - Enero 2018
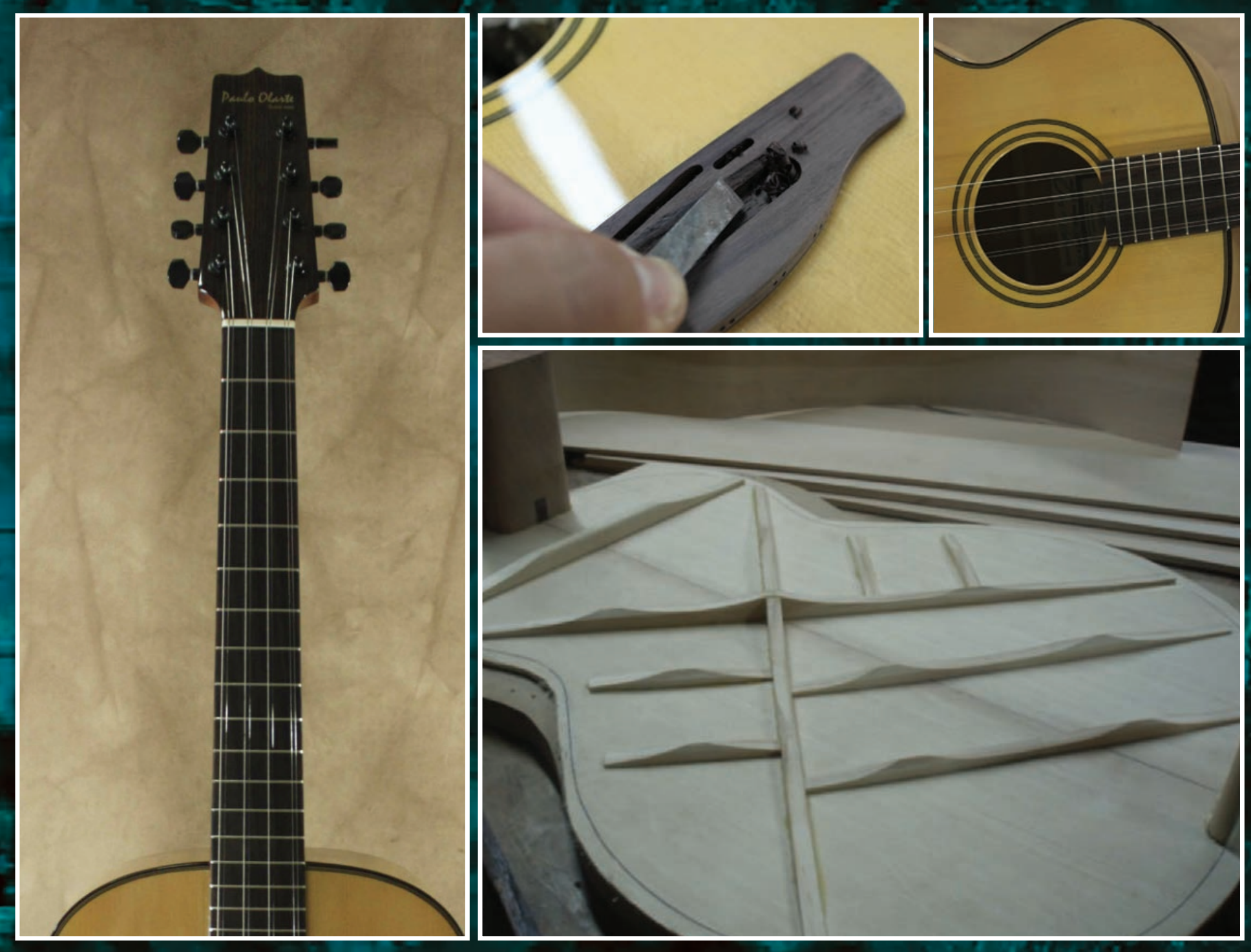


\section{La evaluación formativa desde la concepción de Philippe Perrenoud: una \\ experiencia en la clase de Historia de la Música} The Formative Evaluation from the conception of Philippe Perrenoud: An experience in the History of Music class

Por: Boris Alfonso Salinas Arias ${ }^{1}$

Artículo de reflexión ${ }^{2}$

Recibido: 15 de noviembre del 2016

Aceptado: 15 de junio del 2017

Para citar este artículo/To reference this article Salinas, B. (2017). La evaluación formativa desde la concepción de Philipe Perrenoud: una experiencia en la clase de Historia de la Música. Música, Cultura y Pensamiento, 7 (7), 36-47.

1 Licenciado en Música del Conservatorio del Tolima. Magíster en Educación de la Universidad del Tolima, trabaja en diferentes proyectos de tipo social y comunitario de la Corporación IMA. basalinas@ut.edu.co.

2 Este artículo fue escrito durante el semestre A del 2015; sus reflexiones, críticas, análisis y conclusiones son producto del seminario de evaluación dirigido por la doctora Luz Stella García, curso dictado en el marco de la Maestría en Educación, Modalidad Investigación, ofrecido por la Universidad del Tolima.

\section{Resumen}

Este artículo ofrece una aproximación a los postulados de Philippe Perrenoud, que plantea una mirada hacia la evaluación formativa, marco base para este trabajo. Se hace un ejercicio descriptivo de los instrumentos de evaluación utilizados en la asignatura Historia de la Música $I$, en las carreras profesionales ofrecidas en el Conservatorio del Tolima, para luego llevar a cabo una experiencia sobre la evaluación formativa de Perrenoud durante ocho semanas. A medida que se describe la experiencia, se resaltan las observaciones realizadas a los estudiantes, los instrumentos de evaluación, las reflexiones, las críticas y los comentarios tales como la disposición del estudiante ante un instrumento, la incorporación de la creatividad como elemento evaluable, el cambio actitudinal de los estudiantes y demás. Finalmente, se concluye con respecto a los aportes, pros y contras de la evaluación formativa en la asignatura, los cambios registrados por los estudiantes, el compromiso y el esfuerzo que envuelve al docente.

Palabras clave: historia, música, evaluación formativa, creatividad, observación. 


\begin{abstract}
This article offers an approach to the postulates of Philippe Perrenoud, who proposes a review of the formative evaluation, which is the framework for this work. A descriptive exercise is carried out of the evaluation instruments used in the History of Music I course studied in the professional degree offered at the Tolima Conservatory, in order to then make an experience about the formative evaluation of Perrenoud over an eight week period. As the experience is described, observations are made to the students, including assessment tools, reflections, criticisms and comments such as the student's disposition towards an instrument, the incorporation of creativity as an evaluable element, the attitudinal change of the students, among others. Finally the conclusions are made regarding the contributions, the pros and cons of the subject's formative evaluation, the changes registered by the students, as well as the commitment and effort which also involves the teacher.
\end{abstract}

Keywords: history, music, formative assessment, creativity, observation.

\section{Marco teórico}

El teórico de la evaluación base para este trabajo es el docente e investigador Philippe Perrenoud (2008), su pensamiento se desarrolla en el libro La evaluación de los alumnos: de la producción de la excelencia a la regulación de los aprendizajes. Entre dos lógicas. Allí no hay un modelo ideal de evaluación sino una crítica profunda donde se discuten dilemas como ¿la evaluación está al servicio de la selección o al servicio del aprendizaje? ¿La evaluación es la base para clasificar, calificar, descalificar, segregar y aprobar al estudiante? Y, por tal, ¿da origen a la excelencia y el éxito escolar? A continuación se discutirán algunas de las ideas de Perrenoud que constituyen su propuesta base de pensar una evaluación con carácter formativo.

\section{¿Qué es el fracaso y el éxito escolar?}

Para Perrenoud (2008), el fracaso y el éxito escolar son realidades construidas socialmente, es la escuela quien las crea, les da fuerza de ley, ella certifica, selecciona, da decisiones de orientación. El estudiante fracasa o tiene éxito debido a que se le evalúa. Con la evaluación se crean normas, jerarquías e indicadores, las pruebas Saber -más conocidas como Icfes-, por ejemplo, segregan a los estudiantes dejando por fuera de la universidad pública a quienes no alcanzan un puntaje. Las prácticas de evaluación son aplicadas según la arbitrariedad del docente, instituciones y entes gubernamentales quienes establecen y aplican su concepto de excelencia. Entonces, ¿qué es la excelencia?

La evaluación busca medir y certificar la apropiación de un currículum, el cual está fragmentado por áreas de conocimiento, disciplinas y materias. Los textos legislativos señalan lo que se debe enseñar mas no lo que los alumnos están en condiciones de aprender y los programas le dan un margen amplio al docente, quien aplica su propio concepto de cultura y excelencia (Perrenoud, 2008). Ocurre, entonces, que en la escuela no se enseña todo lo que se evalúa ni se evalúa todo lo que se enseña, incluso no se enseña todo lo que figura en el programa. Muchas veces, lo que se evalúa es débilmente enseñado y resulta de aprendizajes obtenidos fuera de la escuela. En síntesis, no se evalúa lo que se cree evaluar. Todo esto hace que el concepto de excelencia varíe de una institución a otra, de profesor, año lectivo, clase, pero también del gobierno de turno ${ }^{3}$ y su política educativa -y evaluativa - de Estado; en realidad, se reduce a excelente a todo estudiante que obtiene buenas calificaciones construyendo a la vez su antagónico indeseable identificado por Perrenoud como el fracaso escolar.

\section{¿Qué ocurre con los estudiantes frente al fracaso y el éxito escolar?}

Para Perrenoud (2008), en la educación obligatoria hay estudiantes que no entran en competencia por la excelencia, no les interesa o no les importa; muchos no se esfuerzan porque dicen que entre más

\footnotetext{
3 En el caso de Colombia las políticas educativas, por lo general, han sido aisladas y transitorias ya que la prioridad del Estado se ha centrado en la guerra -durante décadas- dejándole a la educación un papel no protagónico.
} 
entregan más les exigen y más esperan de ellos. Otros estudiantes dosifican sus fuerzas en función de las necesidades del momento y no se esfuerzan si no consideran que esto no les traerá algún beneficio, preguntan en clase si el tema que se está explicando aparecerá en la evaluación y de acuerdo con esto toman nota o deciden ignorar la clase. Hay estudiantes que no dan todo su potencial por evitar el matoneo de sus compañeros. Hay adolescentes a los que ni siquiera les importa ser buenos académicamente pues la escuela para ellos ya perdió el sentido, lo cual indica que el problema no es de conocimiento sino falta de actitud. Entonces, ¿quién puede asegurar que alguien que según la escuela es un fracaso, en la vida real sea una persona excelente o viceversa? ¿No pueden ser tachados de falsos los juicios de valor sobre excelencia y fracaso escolar? ¿Cómo saber si un estudiante brinda su capacidad plena o transforma el trabajo escolar en una rutina de memorización sin usar el razonamiento y la intuición pues esto -la memoria- se convierte en la facultad más importante en la mayoría de las evaluaciones aplicadas en la escuela?

Teóricos como Gardner (2011) han ilustrado las diferentes habilidades o inteligencias que poseen las personas que van desde las culinarias hasta las artes como la danza. Aun así, pese a entender que las formas de aprendizaje y las inteligencias son diferentes, se aplican las mismas pruebas evaluativas a todos, se estandariza la evaluación, como si se quisiera el mismo resultado de todos. Una equidad a la hora de realizar la prueba que crea una desigualdad en la enseñanza, pues todos no aprenden igual, todos no poseen las mismas inteligencias y habilidades.

Si se entiende que el éxito escolar es la obtención de óptimas calificaciones, ¿qué pasa entonces con los jóvenes apasionados por las artes que presentan pruebas de Estado en un país donde estas - las artes- se conciben como costura o relleno, algo sin importancia? ${ }^{4}$ ¿Será que la prueba genérica que se aplica por parte del Estado alcanza a medir las capacidades y el potencial artístico? y si las capacidades artísticas y creativas quedan por fuera de la medición, ¿son las artes un relleno en el currículo de la formación básica y media?

4 Es de resaltar que en Colombia las artes no son evaluadas en las pruebas de Estado Saber 11.
¿Qué tipo de sociedad se desea construir en una comunidad que excluye las artes del currículo escolar y de la evaluación educativa? ¿Son consideradas las artes parte del fracaso escolar? ¿Es correcto estandarizar una evaluación que incluya a toda la población y, a su vez, excluya diversas habilidades que no solo son importantes para la vida sino que nos hacen diferentes, creativos, soñadores y a la vez humanos, felices? Con todos estos dilemas vale la pena preguntar: ¿se podrá cambiar la educación sin cambiar la evaluación?

\section{¿Qué propuesta hace Perrenoud?}

Perrenoud propone una evaluación formativa, concepto acuñado originalmente por Scriven (1967) y madurado por Bloom (1971), pero que ha sido enriquecido a partir de los diferentes teóricos como Cardinet (1977), quien define a una evaluación como formativa si contribuye a la regulación de los aprendizajes del curso, con el ánimo de mejorar el aprendizaje de los alumnos, es decir, la evaluación es formativa si ayuda al alumno a aprender. Perrenoud (2008) cita a Astolfi con la frase vuestro error me interesa (p. 15), pues para él la evaluación formativa no es la que sanciona los errores sino que se esfuerza por comprenderlos y trabajarlos. El docente debe transformarse en un investigador en el aula, un investigador de tipo etnográfico que hace su trabajo in situ, que utiliza una serie de instrumentos, procedimientos y actividades que permiten observar cómo funciona el proceso de aprendizaje de los saberes.

La evaluación formativa replantea la forma de enseñar los saberes y de comprender por qué y cómo se produce el error; por tanto, la evaluación se une a la didáctica y se convierte en una herramienta para el aprendizaje del alumno y no en una forma de clasificarlo y segregarlo. Por lo anterior, Perrenoud (2009) plantea pensar en la evaluación como una observación formativa, transformándola no en un modelo ideal sino en una concepción filosófica, que entiende al docente como un investigador que a diario hace un trabajo de campo que le brinda una información valiosa, que de saberse interpretar y analizar, ayudará a mejorar la educación.

\section{Contextualización}

Una vez estudiados y revisados los distintos elementos de la evaluación formativa planteada por Perrenoud (2009), se vivió una experiencia de trabajo de campo en el Conservatorio del Tolima -institución de 110 años de existencia- en los programas 
de pregrado de Licenciatura en Música y Maestro en Música, en una materia común denominada Historia de la Música I. Allí, el semestre dura dieciséis semanas y está dividido en tres cortes, los dos primeros de cinco semanas y el último de seis semanas. Al final de cada corte el docente tiene que subir las notas a la plataforma. El ejercicio se inició en el primer corte académico, durante la segunda semana de febrero del 2015 y finalizó la segunda semana de junio del mismo año. En el primer mes de trabajo se hizo una observación de tipo autoetnográfico que funcionó como diagnóstico, así como un análisis del Plan de Curso y del Plan Educativo Institucional (PEI) del 2015 de la institución. Con esto, se buscó identificar cómo se llevaba a cabo la evaluación y se encontró lo siguiente:

Los instrumentos utilizados para evaluar medían datos exactos en términos históricos que necesitan como principal habilidad la memorización, por lo general, estas pruebas no admiten reflexión ni opinión, aunque el currículo busca desarrollar las habilidades de expresión oral, comprensión lectora y contextualización; los instrumentos y rúbricas son escasos, se usan pruebas escritas las cuales son denominadas como quiz, parcial y examen escrito; la evaluación no es construida de manera colegiada, cada docente fabrica su propia evaluación; no se plantea para el aprendizaje sino para la medición-calificación y aprobación del curso; los estudiantes están más preocupados por establecer porcentajes de notas que en discutir el tipo de evaluación. El desconocimiento arranca por el mismo docente (en este caso quien escribe), que apenas deduce qué es la evaluación sin comprender todos los elementos que están en juego.

Además, el calendario escolar cerrado se convierte en un obstáculo para la retroalimentación, pues cuando el alumno conoce los resultados de la evaluación escasamente se cuenta con una semana para subir la calificación a la plataforma. El semestre continúa sin tiempo para reflexionar sobre lo anterior, lo que ya pasó queda archivado, petrificado en el mundo virtual donde se construye la hoja de vida del estudiante, se pierde la capacidad de modificar o cambiar algo, se convierte en lo inaccesible tanto para el estudiante como para el docente. Subir notas a la plataforma cada cinco semanas es otro mecanismo de estandarización, es una escala de medida en tiempo y nota que representa una forma rígida del currículo, acorde con los sistemas de competitividad, producción y competencias. Asunto álgido de debate pero en el cual está inmerso el sistema educativo colombiano guiado por la educación por competencias y cuyo debate excede las finalidades de este ejercicio.

\section{Cinco principios de la evaluación y su relación con el Conservatorio del Tolima y la cátedra de Historia de la Música I}

A continuación se presentan a modo de diagnóstico, cinco principios de la evaluación educativa propuestos por Santos Guerra (2010), aplicados a las prácticas evaluativas de la asignatura Historia de la Música l:

1. Es preciso que la evaluación sea un proceso participativo: este principio no se cumple, al inicio de semestre se hace un acuerdo pedagógico general en el que poco se habla de la evaluación, la atención se centra en la calificación, los porcentajes de las notas y su equivalencia en la nota definitiva, así como en la hora de entrada y salida de la clase.

En esta experiencia se observó que los estudiantes están más preocupados por establecer porcentajes de las notas en los que las pruebas escritas no tengan mucho valor más que en discutir el tipo de evaluación, instrumento o método a emplear. El desconocimiento arranca por el mismo docente (en este caso quien escribe), que apenas deduce qué es la evaluación sin comprender todos los elementos que la integran. Así mismo, la participación del estudiante en este asunto es nula.

2. Para que la evaluación tenga rigor ha de utilizar instrumentos de evaluación diversos: los instrumentos que se utilizan son escasos, pruebas escritas que en su mayoría están recargadas de preguntas de tipo memorístico, y que se denominan con múltiples nombres como quiz, parcial y examen escrito, que en síntesis son lo mismo. Se plantean algunas habilidades que deben ser evaluadas como la expresión oral, la capacidad de comprender y explicar diversos elementos de la historia, pero se nota la ausencia de instrumentos que permitan medir esas habilidades como foros, ponencias y contraponencias, discursos, elaboración de escritos, etc. Así mismo, aparecen algunas actividades enunciadas 
como mesas redondas y actividades de audición musical pero sin estar ligadas a las habilidades $u$ objetivos que pretenden medir, es decir, hay una desconexión entre el objetivo a alcanzar y el instrumento para medir si se alcanzó ese objetivo.

3. La evaluación tiene que servir para el aprendizaje: como tal, la evaluación no se plantea para el aprendizaje sino para la medición-calificación y aprobación del curso, no hay enunciados que formulen la necesidad de aprender de la evaluación ni por parte del docente ni del estudiante. El mismo sistema que se maneja en el conservatorio de calendario cerrado limita aprender de la evaluación, hay poca retroalimentación pues cuando un estudiante sabe cuál es su nota de corte, esta se vuelve definitiva por lo que no hay tiempo para hacer correcciones o mejoras, todo queda en un pasado que el estudiante debe superar rápidamente para ahora concentrarse en el nuevo corte académico. Sin embargo, el hecho de que la nota se convierta pronto en definitiva introduce un nivel de exigencia y compromiso adicional con la asignatura por parte del estudiante y del docente.

4. Es importante hacer metaevaluación, evaluar las evaluaciones: este punto es inexistente en el currículo y en la práctica docente personal se hacía más por asegurar la objetividad del instrumento a la hora de poder responder a los reclamos de los estudiantes al decir que su nota no es justa, que por mejorar la evaluación misma, es un asunto complejo pero que parece apenas obvio y es que ¿cómo puede mejorar la evaluación si esta misma no se somete a evaluación? Someter a evaluación las evaluaciones debe ser una práctica incluida en los tiempos y cargas laborales de preparación de clase tanto del docente en su asignatura- como de los docentes en sus reuniones de área.

5. La evaluación debe ser un acto colegiado: en la institución la evaluación tanto institucional como docente se hace de manera individual, sin llegar a un consenso, sin siquiera socializarla con los demás, quizás por miedo a que los demás docentes sepan los métodos personales de evaluación e incluso a que los critiquen, juzguen o copien en caso de que una forma sea más exitosa ante los estudiantes, o también por desco- nocimiento. Ante la disciplina o arte de evaluar, los docentes y la institución se quedan cortos, la evaluación se vuelve una misión individual, quizá deseable pero siempre obligatoria. Poco se habla de ella con los demás docentes. Seguramente habrá elementos propios de la profesión musical que influyen en la manera de construir la evaluación colegiada como, por ejemplo, el ego de todo artista, que le hace buscar los propios métodos sin escuchar o mirar a los demás.

El éxito y el fracaso escolar no solo envuelven al estudiante sino también al docente, están presentes, lo permean e incluyen en su discurso por medio de los procesos de evaluación docente, lo seleccionan y clasifican, convierten la profesión en un ejercicio permanente de competencia $y$, en la competencia, lo importante es llegar primero, ser el mejor. La evaluación docente no solo da continuidad laboral sino que también ofrece mejoras salariales.

\section{Historia de la Música I. Ejercicio de evaluación según los parámetros y críticas de Philippe Perrenoud}

La primera conclusión a la que se llegó al comenzar este ejercicio fue que se debía ver la evaluación como una constante de cada clase y no como algo al final del ciclo. Por tal, se hicieron evaluables diversas actividades $y$, al mismo tiempo, calificables en vista del poco tiempo disponible para subir notas, la pregunta: ¿se debe regular el aprendizaje o la actividad?, aún genera la inquietud de si se hizo y se sigue haciendo lo correcto o no.

El ejercicio se llevó a cabo en la asignatura Historia de la Música I, cuyos temas van desde la música de las antiguas civilizaciones hasta la fase final de la Edad Media. A este curso asisten estudiantes de tercer semestre de los programas Licenciatura en Música y Maestro en Música, dos pregrados de formación musical del Conservatorio del Tolima, que tienen enfoques distintos, siendo el primero de formación en docencia musical y el segundo de formación en interpretación de un instrumento. El curso estaba compuesto por un total de 25 estudiantes con el cual se trabajó en óptimas condiciones físicas y técnicas, pues la institución cuenta con amplios salones, equipos de proyección y sonido, instrumentos musicales, así como una clavinova o piano por salón. 
Es de resaltar que las clases se hicieron en la sede tradicional del Conservatorio del Tolima, edificación declarada monumento nacional de Colombia mediante la Ley 112 de 1994, lo cual le da un plus invaluable a la asignatura y a la institución como universidad musical. A continuación se relata la experiencia de evaluación formativa que fue implementada en la asignatura mencionada desde el segundo corte -semana seis de clases- hasta el final del semestre -semana dieciséis-.

\section{Primera semana de la experiencia: semana} seis de clase

Como en el corte anterior se habían trabajado temas sobre la música de las antiguas civilizaciones, se decidió dejar una tarea creativa para verificar qué tanto se había asimilado la información del tema visto, por lo cual debían realizar una composición ceñida a la cultura que por grupos ya habían trabajado y hacer la muestra de esa obra musical en clase. Los resultados fueron interesantes, los grupos hicieron una muestra musical de buen nivel artístico, dos de los grupos asistieron uniformados con ropas y uno de ellos hizo una clara alusión al país que representaba, la India. Los grupos no solo asimilaron la información musical de cada cultura sino que pudieron crear una obra musical de acuerdo con esta información, los estudiantes manifestaron estar satisfechos con la actividad, la cual les permitió poner en práctica los conceptos teóricos que habían visto. Así mismo, se disfrutó una materia que de por sí puede resultar densa.

\section{Segunda semana de la experiencia: semana siete de clase}

En principio, la clase consistió en explicar cómo funciona el sistema musical de la Edad Media, para lo cual debían realizar una composición musical breve individual y entregarla en la partitura para la siguiente sesión. Luego, se empezó a trabajar un taller en clase con base en lecturas previas. Las preguntas eran abiertas en temas complejos como por ejemplo: ¿qué relación hay entre música y religión?, así como la elaboración de cuadros comparativos que les permitieran organizar la información. El taller fue resuelto en parejas $y$, mientras tanto, como docente, hacía una observación a los grupos en cuanto a lo actitudinal y la manera en que trabajaban en la clase; la mayoría había leído el material, otros tomaron los temas principales y buscaron en otras fuentes, pocos no habían leído y, en consecuencia, se sentían en desventaja. La reacción tomada como docente ante los estudiantes que no habían leído fue precisar que la distribución correcta y organizada del tiempo puede hacer que tengan un buen rendimiento sin necesidad de trasnochar y correr al final de un periodo pues, de cierta manera, no hacer las cosas con tiempo lo único que hace es acumular el trabajo. A continuación se presentan apartes del taller:

1. ¿Qué rol cumplen las tradiciones religiosas en la conformación de las músicas del primer milenio después de Cristo?

2. Realice un cuadro comparativo donde manifieste el tipo de culto religioso, pueblo practicante, idioma hablado, características musicales y otras aspectos que considere importantes, del primer milenio después de Cristo.

3. ¿Está la música actual ligada a un culto religioso? ¿Cuáles pueden ser las razones que explican este fenómeno?

4. Realice un cuadro donde establezca los aportes de Guido de Arezzo.

5. Realice una cronología donde explique la evolución de la notación musical.

6. ¿En qué consiste la liturgia romana?

7. ¿Cuál es la estructura de la misa y el uso de la música en esta?

8. Mencione las principales características del canto llano.

9. Realice un cuadro comparativo con los géneros y formas del canto.

10. Escriba acerca de los músicos de la época (Taller de Historia de la Música I, Salinas, 2015).

Para desarrollar el taller, algunos chicos acudían a Internet por medio de sus celulares y otros discutían sobre las preguntas abiertas, principalmente, de tipo religioso. Se pudo observar que gran parte de los estudiantes pertenecen o pertenecieron a alguna iglesia cristiana por lo cual la pregunta que inquiere sobre la relación música-religión les Ilamó mucho la atención, de cierta manera, los llevó al contexto de su vida cotidiana. Muchos se iniciaron en la música 
gracias a iglesias cristianas y algunos reconocieron que parte de su incursión en la religión fue gracias a la música. De aquí se puede decir que la evaluación no solo ayuda a saber si un estudiante ha preparado o no su tarea, sino también, que se pueden reconocer e identificar factores que son importantes en la vida de los estudiantes, como que conocieron la música gracias a la religión y a su vez fortalecieron el lazo religioso guiados por el gusto musical.

Se podría pensar que el legado religioso de la música de Europa central sigue vigente, unido desde la Edad Media a la fe cristiana. Sin embargo, no puede ser solo legado el identificar a la religión como un indicador de iniciación musical, pues esto puede sugerir distintas hipótesis como que los estudiantes no son apoyados en sus inicios musicales por sus padres, o que no tienen un ambiente familiar cultural rico en temas musicales. También, se podría pensar que el acercamiento musical en la escuela es pobre o nulo, ya que el sistema educativo colombiano no da la oportunidad de una formación artística musical que sea laica, propia de un Estado de derecho de una nación no confesional y a su vez multicultural.

\section{Tercera semana de la experiencia: semana ocho de clase}

Para la socialización del taller, cada grupo formuló tres preguntas; al azar, uno de los grupos debía responderla y se daba la oportunidad para que otro grupo le ayudara en caso de no tener la respuesta; al final se recogió la opinión de todos sobre la actividad $y$ coincidieron en que era agradable poder discutir diferentes temas, hablar de cómo se utilizó la música como elemento de poder en diferentes culturas y momentos de la historia, y de cómo en la actualidad se sigue haciendo. Además, los estudiantes manifestaron el acierto de socializar las respuestas y discutirlas, no solo entregar un trabajo sin saber si lo habían hecho bien o mal.

La mayoría de las preguntas que formularon los chicos eran puntuales y memorísticas, como por ejemplo: "indique cómo se llama el personaje que hizo tal cosa, cuál fue el aporte más importante de..., en qué año pasó tal cosa...", en esas ocasiones se les sugirió construir preguntas que llamaran a la reflexión, como: ¿por qué creen que la música es una herramienta de la religión?, ¿cuál fue el papel de los músicos durante la Edad Media?, ¿de qué y cómo vivía un músico en la Europa medieval? Después de socializar el taller se proyectó un documental que explicaba la evolución de la notación musical, en esta algunos estudiantes se dispersaron con los celulares. Al final de la clase se recogieron los informes del taller y los ejercicios de composición que había dejado de tarea la semana anterior.

\section{Cuarta semana de la experiencia: semana nueve de clase}

En esta clase se hizo una prueba escrita de cinco preguntas abiertas que dieron espacio para el debate $y$ la reflexión. Se observó que el estudiante se indispone y cambia de actitud en clase cuando se enfrenta a una prueba escrita; particularmente, los estudiantes son ansiosos, pues a medida que avanza el tiempo y al ver que los demás entregan el examen, comienzan con afán a preguntar la hora y a escribir rápido para entregar lo más pronto posible. La mayoría de los estudiantes presentó la prueba en menos de 70 minutos pese a que se tenían destinadas dos horas para esta. Al final del examen se incluyó una autoevaluación guiada y se dio la opción de que cada estudiante anexara puntos que considerara convenientes.

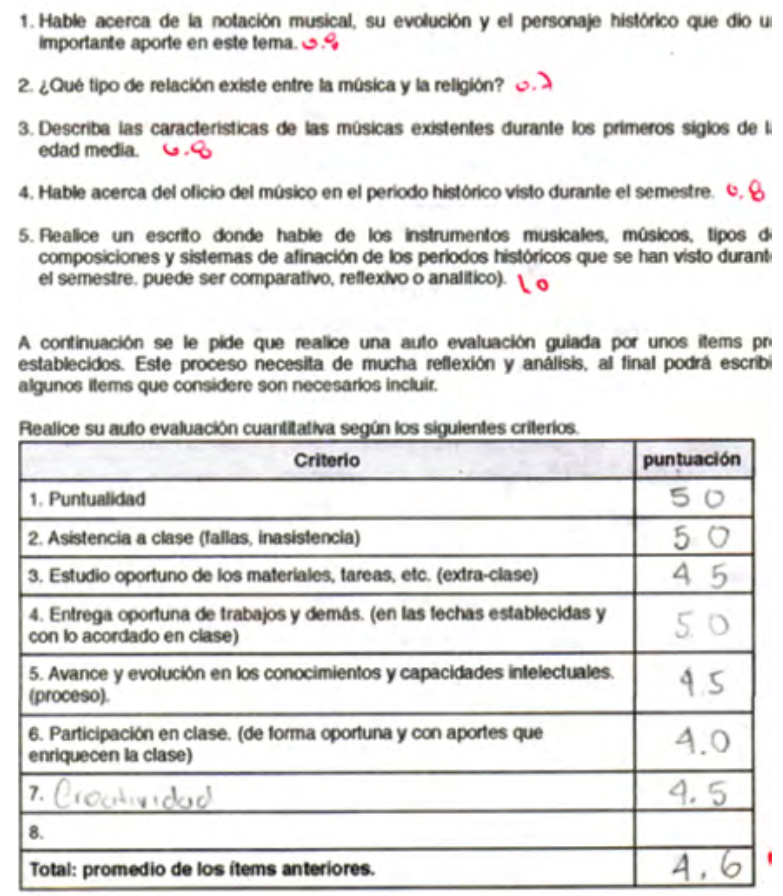

Figura 1. Instrumento de evaluación escrita y autoevaluación empleado en la asignatura Historia de la Música I

Fuente: el autor (2015). 
Al analizar las respuestas se encontró que muchos no leen bien las preguntas, esto coincide con otros cursos en los que, por ejemplo, al incluir preguntas erradas, no se dan cuenta de los errores que tienen la misma formulación. También se encontró que, si bien las preguntas son abiertas, cerca de la mitad de los estudiantes dan respuestas demasiado concretas poco abiertas a la discusión. Los estudiantes están muy acostumbrados a las preguntas de memoria e incluso se sienten más cómodos este tipo de examen. Al preguntar en clase por qué entregaron los exámenes sin utilizar todo el tiempo que disponían, se corroboró con el diálogo que era un asunto de ansiedad. Manejar la ansiedad es una habilidad que no se enseña en clase y es una habilidad fundamental para los músicos, no solo para responder mejor una prueba, sino para el desarrollo de la vida artística en los distintos escenarios en los que un músico participa.

En las autoevaluaciones pocos anexaron ítems para la calificación, los que incluyeron ítems nuevos no tenían una calificación buena en los anteriores y esos puntos adicionales respondían a actitudes de clase, como respeto por los compañeros. Una estudiante adicionó el ítem creatividad, bastante acertado teniendo en cuenta que dos de las actividades del curso tenían implícita esta habilidad, que es fundamental para cualquier artista, no obstante, fue la única estudiante que lo hizo.

Después de la clase se revisó el trabajo de composición, una pequeña obra musical inspirada en la era medieval. La sorpresa fue grata, no solo hicieron la tarea sino que la belleza estética de los ejercicios fue de un valor especial, en este sentido, se trató de escribir en cada una de las obras recomendaciones para hacer que sonaran mejor, aunque ese ejercicio es completamente habitual en el quehacer musical pero se debe resaltar que es acorde con el discurso de la evaluación formativa y no se había aplicado en la clase de Historia.

Score

El Timbre

Guabina

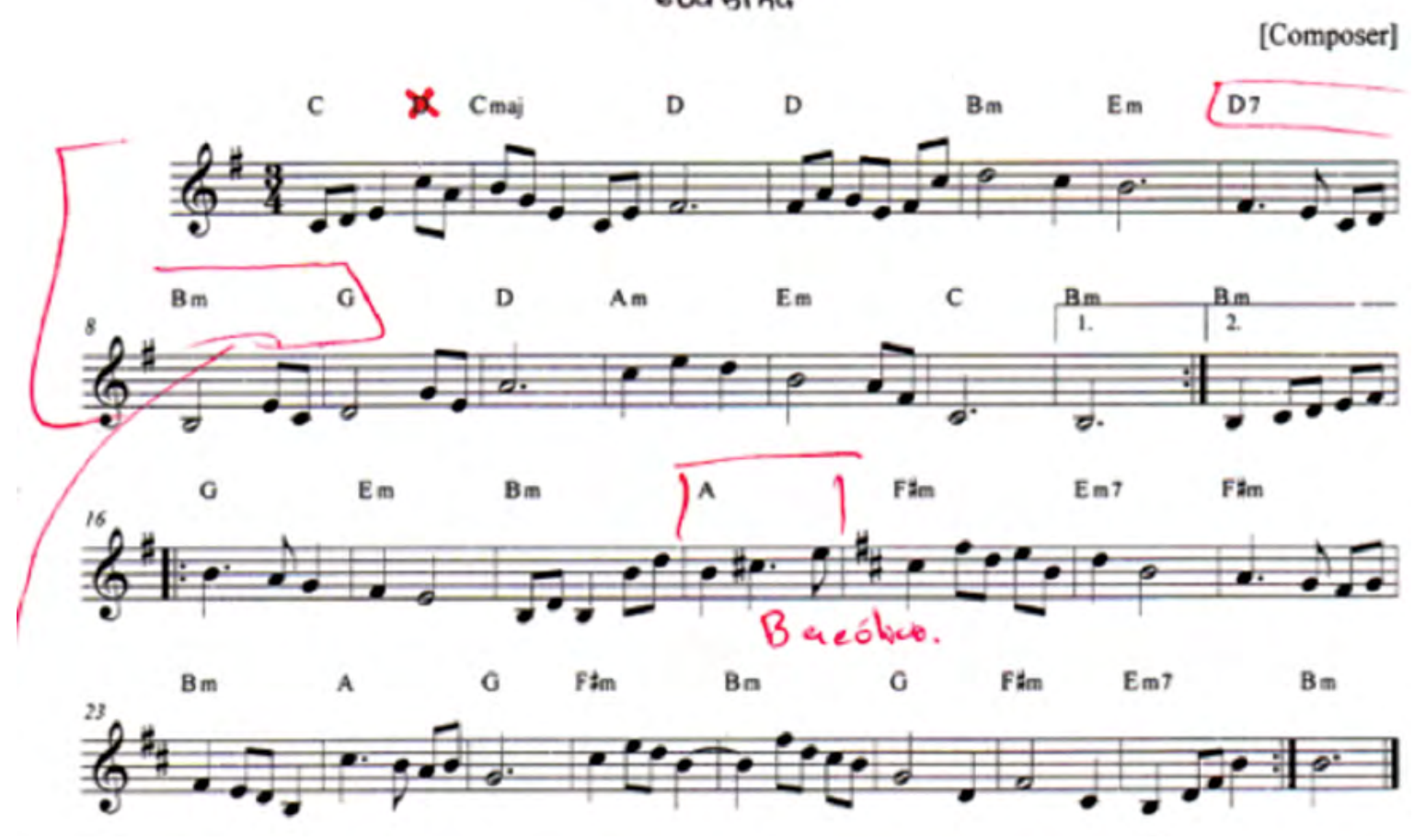

Figura 2. Ejercicio de composición de una estudiante de la clase Historia de la Música l, 2015

Fuente: el autor (2015). 
El deseo de implementar el ejercicio de composición no fue ceñirse a las reglas estéticas y crear de forma específica en un estilo determinado, sino incentivar la creatividad de los estudiantes buscando utilizar los elementos históricos de la música como recursos para la creación musical. Esto se hizo en un discurso que busca que la historia no solo se quede en el pasado sino que sirva para proyectar el presente, comprender que la historia es conocimiento y como conocimiento se puede utilizar en cualquier actividad propuesta. El último ejercicio fue que cada estudiante evaluara el curso de manera anónima y libre, se observó que a escala general sienten que aprenden más cuando ponen en práctica y sienten la libertad de componer música a partir de lo visto en clase. Así mismo, hacen sugerencias para poder replantear el espacio donde se desarrolla la clase, hacer algunas salidas a museos o prácticas de campo y un mayor compromiso personal -como estudiantes- del curso.

\section{De la quinta hasta la décima semana de la experiencia: semana diez a la dieciséis de clase}

En el tercer corte, ante los buenos resultados, se incorporó como parte integral del curso el componente de creatividad para poder asimilar mejor las texturas musicales de cada momento histórico. Para adentrarse en la música de la Edad Media se construyó un extenso taller en el que los estudiantes debían hacer ejercicios creativos musicales; para ello se llevaron a clase diversos ejemplos de artistas que hacen música de esta época. En el taller se introdujeron preguntas que ayudaban a clasificar y a desnudar las técnicas utilizadas en este periodo histórico para culminar con un ejercicio creativo en grupos, en el que debían escribir la partitura e interpretarla en clase. El taller incluyó como guía páginas web que tuvieran texto, videos y audios en los que se explicaran las características de la música de la Edad Media, se entregó una lista de bibliografía disponible en la biblioteca del conservatorio y se entregó material digital. A continuación se anexan las preguntas del taller.

\section{Taller de Historia de la Música I Tercer corte \\ Docente: Boris A. Salinas Arias}

1. ¿Qué relación hay entre música y poesía durante la Edad Media? ¿Existen vínculos en la actualidad de esa relación?

2. Realice un escrito de media página acerca de los trovadores, troveros y juglares.

3. Mencione a nivel general las características de la monodia religiosa latina.

4. Realice un ejercicio de composición monódica acorde con lo visto en la unidad II, este ejercicio no necesita ser escrito pero debe ser interpretado en clase.

5. ¿En qué consiste la polifonía primitiva, que técnicas utiliza y cuáles son los intervalos considerados consonantes y disonantes?

6. Realice un ejercicio de composición medieval polifónico teniendo en cuenta las características técnicas tratadas en la unidad III. Este ejercicio debe ser escrito en partitura e interpretado en clase.

7. ¿En qué consiste el ars Nova y el ars Antiqua?

8. Realice un escrito de opinión sobre el tema La sombra de Pitágoras. Armonía, composición, ciencia y religión en la música medieval, cuyo enlace se encuentra en la página. https://bustena.wordpress.com/2013/09/16/la-sombra-de-pitagoras-armonia-composicion-ciencia-y-religion-en-la-musica-medieval/

9. Realice una composición teniendo en cuenta las características de la polifonía de los siglos xIII y xIV.

10. Haga un cuadro comparativo de los estilos compositivos tratados en este taller, donde pueda establecer similitudes y diferencias de las técnicas, acordes, texturas y otros aspectos que consideren sean importantes para comprender la música de este periodo.

11. Realice un cuadro donde pueda comparar los diversos compositores, lugar de procedencia, año en que vivió, estilo musical y aportes de los periodos tratados en este taller. 
La actividad será socializada en clase (esto será una nota de la actividad), se entregará por escrito y en la clase siguiente se realizará la interpretación-socialización de los trabajos creativos. (Taller de Historia de la Música I, Salinas, 2015).

Al hacer la evaluación de los trabajos se notó responsabilidad en la elaboración del taller, los trabajos creativos contaron con alto nivel de compromiso e imaginación, todos variados en complejidad y belleza, con un valor artístico. Un grupo hizo una obra teatral de títeres en la que se contaba la historia de un caballero, un dragón y una princesa, musicalizado en el contexto medieval; otro grupo hizo una mezcla entre rock y música antigua manipulando creativamente las sonoridades medievales. Incluso, una chica que no pudo ensayar con ningún grupo hizo sola su trabajo y les pidió colaboración a otros estudiantes para que la apoyaron en su muestra.

La calidad de las presentaciones estuvo a la altura de cualquier muestra musical, los estudiantes se sintieron satisfechos de este tipo de evaluación y manifestaron que era mejor que el parcial escrito. Es de anotar que ante este tipo de evaluación hay muchos componentes adicionales que hacen parte del aprendizaje, como el trabajo en grupo, la empatía, la creatividad, la comprensión del momento histórico y su proyección en la actualidad, la capacidad de aprender rápidamente la música y los textos, así como imprimir una intención para tocar en público, la puesta en escena, la interpretación. Nótese que todos estos criterios de evaluación son difíciles de medir en un instrumento escrito.

\section{Conclusiones}

Hacer un cambio de la evaluación no es tarea fácil, pero el solo hecho de intentarlo mejora la actividad docente. La evaluación en este sentido sirve para recolectar información y entender cómo funciona el aprendizaje de los estudiantes, ayuda a tomar mejores decisiones con las actividades así como a reflexionar sobre los conocimientos que debería brindar la escuela o la universidad, como el manejo de la ansiedad y saber distribuir el tiempo en una prueba escrita. También se constató que este ejercicio consume más tiempo y más energía que una clase y evaluación tradicional, da mejores resultados pero demanda más esfuerzo, estudio y creatividad, más didáctica y, por lo mismo, más preparación.
El número de fallas por estudiante bajó, al pedirles a los estudiantes que hagan una evaluación del curso por escrito ellos manifestaron mayor satisfacción de estar en clase y son autocríticos al formular que deben ser más comprometidos; también se despierta más la participación de los estudiantes, aunque se debe seguir trabajando en este aspecto. Como experimento de clase, no se llamó a lista ni se pusieron fallas durante el semestre, esto como barómetro que permitiera establecer si por interés propio los estudiantes llegaban puntuales. Lo que ocurrió fue que en el primer corte casi todos llegaban a las 7:20 a. m. -la clase era a las 7:00 a. m.-, pero en el segundo corte los estudiantes fueron más puntuales. Desde luego, este dato no es contundente, pero permite pensar la asistencia y puntualidad de los estudiantes como una forma de medir qué tan emocionante es una clase y si despierta el deseo de aprender. La hipótesis es: si no existe un condicionante y la clase es emocionante, el estudiante no debería llegar tarde.

Por otro lado, al hacer los ejercicios creativos los estudiantes no solo los hacen sino que disfrutan del aprendizaje, son capaces de plasmar estéticamente las características de la época y reproducirlas a la hora de crear música. El aprendizaje en este caso alcanzó un nivel significativo. El reto aquí es poder seguir creando situaciones de aprendizaje que incorporen la creatividad y articularlas con los contenidos de los diversos cursos, arriesgarse a probar nuevas actividades y esto obviamente incluye el equivocarse, tener la capacidad de arriesgar y evaluar las actividades para aprender del error. Aprender del error, como lo entienden Anijovich y González (2011), es encontrar la oportunidad en la equivocación y el fracaso, el músico se enfrenta cotidianamente con una fobia al fracaso artístico y personal, pues se vive a la sombra de los grandes músicos de la historia: la sombra de Bach, Mozart, Beethoven, Chopin y muchos otros que hicieron lo que pudieron en su momento pero que son idolatrados como figuras cuasimíticas que habitan en el Olimpo, un Partenón de la música.

Se configura una visión perfeccionista de la música en la que el error es sinónimo de fracaso mas no de oportunidad, esto se extiende y contamina la educación musical, se genera como fenómeno y se configura y perpetúa como parte del currículo oculto. Aprender del error abarca un cambio de la menta- 
lidad perfeccionista y condenatoria típica del fanatismo religioso, comprende una liberación ideológica del imperio del ego y de la frustración artística. Con todo esto, lograr un cambio en la concepción que tienen los músicos del error no es tarea fácil. ¿Cuántos músicos asisten a un concierto sin estar pendientes de los errores de interpretación y centrados en la belleza de las obras? ¿Cuántos músicos se suben a un escenario sin miedo a equivocarse y a ser juzgados por sus colegas por los errores que puedan cometer en el concierto?

Los músicos son profesionales que buscan un alto grado de desempeño en su oficio, lo que los lleva a tener un alto nivel de autoexigencia que puede desembocar en estrés, frustración y depresión al no alcanzar el ideal planteado. Este fenómeno está presente no solo en la parte interpretativa del instrumento sino en todo el campo laboral del músico. El músico se alimenta del aplauso del público y, cuando no alcanza este reconocimiento, tiende a sentir y pensar que no se ha hecho bien la labor. He ahí la importancia de cambiar el concepto del error.

Si se cambia el concepto de error como fracaso y se le entiende como oportunidad, se podría ver que es una constante del proceso de enseñanza-aprendizaje, brinda la oportunidad de identificar las dificultades y buscar una posible solución, da la oportunidad de fortalecer la autoestima y seguridad del músico, de mejorar la formulación y organización del currículo, de crecer como profesores, estudiantes, artistas y seres humanos.

Finalmente, se debe concluir que la evaluación permite observar, diagnosticar y sacar a la luz los diversos fenómenos y problemas que existen en la cotidianidad académica y en la misma vida, tanto de los estudiantes como de los docentes, demanda más trabajo y compromiso, más ojo de investigador, más preparación y mayor compromiso docente, aun así Perrenoud -aludiendo a las protestas estudiantiles del 68 - convoca a todos los maestros con la frase seamos realistas, pidamos lo imposible (Perrenoud, 2008, p. 89).

\section{Referencias}

Anijovich, R. y González, C. (2011). Evaluar para aprender, conceptos e instrumentos. Buenos Aires: Aique Educación.

Altet, M., Paquay, L. y Perrenoud, Ph. (dir.) (2003). A profissionalização dos formadores de profes- sores. Porto Alegre: Artmed Editora (trad. de M. Altet, L. Paquay, L. y Ph. Perrenoud (dir.) Formateurs d'enseignants. Quelle professionnalisation? Bruselas: De Boeck).

Bloom, B. S., Hastings, J. y Madaus, G. F. (1971). Handbook in formative and summative evaluation of student learning. New York: McGraw-Hill.

Cardinet, J. (1977). Objectifs pédagogiques et évaluation individualisée. Neuchâtel: Institut romand de recherches et de documentation pédagogiques.

Conservatorio del Tolima. (2014). Mallas y planes curriculares, programa Licenciatura en Música. Ibagué: Facultad de Educación y Artes Conservatorio del Tolima.

Conservatorio del Tolima. (2015). Plan de Curso por Competencias, programa Licenciatura en Música, plan 2012. Ibagué: Facultad de Educación y Artes Conservatorio del Tolima.

Conservatorio del Tolima. (2015). Documentos y evaluaciones realizadas en la clase Historia de la Música I durante el semestre A de 2015. Ibagué: Facultad de Educación y Artes Conservatorio del Tolima.

Gardner, H. (2011) Mentes creativas: una anatomía de la creatividad. Barcelona: Paidós.

Perrenoud, Ph. (2002). A prática reflexiva no ofício de professor: profissionalização e razão pedagógicas. Porto Alegre: Artmed Editora (trad. en portugais de Développer la pratique réflexive dans le métier d'enseignant. Professionnalisation et raison pédagogique. París: ESF).

Perrenoud, Ph. (2004). Os ciclos de aprendizagem. Um caminho para combater o fracasso escolar. Porto Alegre: Artmed Editora (trad. en portugais de Les cycles d'apprentissage. Une autre organisation du travail pour combattre l'échec scolaire. Sainte-Foy: Presses de l'Université du Québec).

Perrenoud, Ph. (2008). La evaluación de los alumnos: de la producción de la excelencia a la regulación de los aprendizajes. Entre dos lógicas. Porto Alegre, Buenos Aires: Alternativa Pedagógica.

Pinar, W. (2014). La teoría del currículo. Madrid: Narcea.

Salinas, B. (2015). Taller de historia de la Música I. Manuscrito no publicado. Ibagué: Conservatorio del Tolima. 
Santos, M. A. (2010). La evaluación como aprendizaje, una flecha en la diana (3. ${ }^{\mathrm{a}}$ ed.). Buenos Aires: Borvin.

Scriven, M. (1967). The Metodology of evaluation. En R.W. Tyler, R.M Gagne, and M. Scriven, Perspetives of curriculum evaluation (Ame- rican Educational Research Monograph on curriculum Evaluation no. 1). Chicago: Rand McNally.

Tyler, R. (1973). Principios básicos del currículo. E. Molina (trad.). Buenos Aires: Troquel. 


\section{Contenido}

VOL. VII N.o 7 - Enero 2018

4 resemancen

\section{Música y Cultura}

Ruana y carranga: dos símbolos campesinos de origen múltiple Leonardo Zambrano Rodríguez

\section{Música y pensamiento}

18 Arte y música como apropiación social del conocimiento en el proyecto regional Aprociencia Humberto Galindo Palma

36

La evaluación formativa desde la concepción de Phillipe Perrenoud: una experiencia en la clase de Historia de la Música Boris Alfonso Salinas Arias

\section{Música en clave}

48

Creación de herramientas de análisis para

el estudio del contrapunto tonal: una aproximación desde la música popular María Cristina Vivas Barrera, Adrián Camilo Ramírez Méndez, Juan Gabriel Alarcón (colaborador)

(1) Tiple solista en Colombia:

Sergio Andrés Camero Tierradentro, Jorge Iván Ramírez Marín, Yordi Andrey Vargas Guzmán

\section{Separata}

Niebla de Chipre para orquesta de cuerdas segundo premio en el Concurso Internacional de Composición "Música Jove",

Valencia-España 2016

Andrés A. Garzón Charry

Convocatoria Revista No. 8

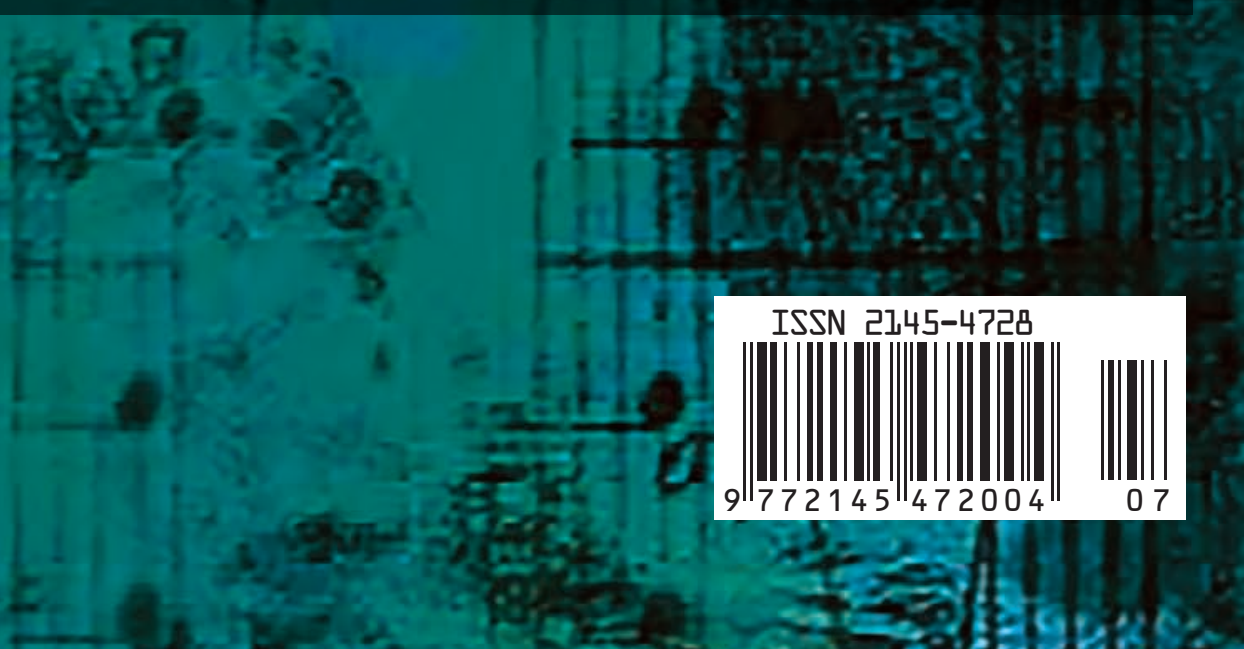

